Introduction The use of non-invasive respiratory support for COVID-19 related respiratory failure outside of Intensive Care Units (ICU), delivered in respiratory support units, became widespread during the COVID-19 pandemic. There is paucity of data thus far for outcomes in this patient cohort.

Methods We retrospectively reviewed the medical notes of 89 patients with COVID-19 pneumonitis admitted to our recently opened (April 2020) Respiratory Support Unit (RSU) at Colchester General Hospital from 17th April, 2020 to 13th February, 2021. Mean age was 69 years (range 30 - 93 years) and 56 patients were male. Sixty three patients received continuous positive airway pressure (CPAP), 6 patients received high flow nasal oxygen (HFNO), 13 patients received a combination of CPAP with periods of HFNO and 7 patients received non-invasive ventilation (NIV).

Results On admission to the RSU, patients had average saturations of $87.5 \%$ (range $50-99 \%$ ) with an average pO2 of $7.69 \mathrm{kPa}$ (range $3.6-18$ ). The majority of patients were receiving fraction of inspired oxygen $(\mathrm{FiO} 2)$ greater than 0.6. RSU success (wean from CPAP/NIV/HFNO) was 24.7\%. RSU failure (either escalation to ICU or death, depending on treatment-escalation status) was $75.3 \%$. The overall mortality rate was $71.9 \%$. Mortality was higher $(80 \%)$ in those patients who were not for escalation to ICU. Mortality in those for full treatment escalation was $42.1 \%$. Higher mortality occurred in patients with multiple comorbidities, increasing age and higher Rookwood Clinical frailty scores (CFS). Patients without any additional organ dysfunction had lower mortality $(62.5 \%$ vs 87.9\%). Increasing mortality was observed with increasing time from hospital arrival to RSU admission. All patients aged 80 years or above and those with a CFS 6 died. Complications included pulmonary embolism $(n=3)$, pneumothorax $(\mathrm{n}=1)$ and pneumomediastinum $(\mathrm{n}=1)$.

\begin{tabular}{|c|c|c|c|c|c|c|}
\hline \multicolumn{7}{|l|}{ Mortality by groups } \\
\hline Age groups & $30-39$ & $40-49$ & $50-59$ & $60-69$ & 70-79 & $\geq 80$ \\
\hline Mortality (\%) & 0 & 40 & 50 & 71.4 & 79.5 & 100 \\
\hline CFS & 1 & 2 & 3 & 4 & 5 & 6 \\
\hline Mortality (\%) & 20 & 43 & 79 & 77.3 & 87.5 & 100 \\
\hline $\begin{array}{l}\text { Number of } \\
\text { comorbidities }\end{array}$ & 0 & 1 & 2 & 3 & 4 & \\
\hline Mortality (\%) & 42.9 & 46.1 & 76.9 & 80 & 84.6 & \\
\hline $\begin{array}{l}\text { Time to admission to } \\
\text { RSU (days) }\end{array}$ & Less than 1 & $1-2$ & $3-7$ & 8 or more & & \\
\hline Mortality (\%) & 63.2 & 64.7 & 81.5 & 81.8 & & \\
\hline
\end{tabular}

Conclusion These data demonstrate the high mortality in patients with respiratory failure secondary to COVID-19 pneumonitis admitted to our RSU, particularly in those who were not for escalation to ICU. Increasing age, number of comorbidities and CFS were associated with treatment failure as well as time between presentation and admission to RSU. Careful patient selection with consideration of these factors is vital when identifying patients appropriate for respiratory support.

\section{P215 A RETROSPECTIVE OBSERVATIONAL STUDY OF COVID- 19 PATIENTS ON A RESPIRATORY HIGH CARE UNIT (RHCU)}

R O'Neill, J Reece, A Lal, M Saeed, SL Tan. Worcestershire Acute Hospitals NHS Trust, Redditch, Worcestershire, UK

\subsection{6/thorax-2021-BTSabstracts.324}

During the COVID-19 pandemic, our district general hospital serving a population of 200,000, transformed a medical ward into a Respiratory High Care Unit (RHCU) - managing COVID positive and negative patients. Our primary mode of oxygenation was continuous positive-airway pressure (CPAP).

Between 1st June 2020 and 31st March 2021, 359 patients were admitted to RHCU. We performed a retrospective observational study assessing COVID-positive aerosol generating procedure (AGP) patients. 156 patients (43\%) fit this criteria, 144 notes were available for analysis.

$96 \%$ of patients were admitted on $\geq 40 \%$ FiO2. $23 \%$ of patients were admitted from Accident and Emergency.

\begin{tabular}{|c|c|}
\hline RHCU data & COVID-19 AGP $(n=144)$ \\
\hline Gender - male (\%) & 72 \\
\hline Age (mean years) & 66.2 \\
\hline White ethnicity (\%) & 94 \\
\hline Current or ex-smoker (\%) & 45 \\
\hline \multicolumn{2}{|l|}{ CFS of admissions (\%) } \\
\hline$-1-2$ & 52 \\
\hline$-3-4$ & 36 \\
\hline$-\geq 5$ & 12 \\
\hline FiO2 $\geq 40 \%$ prior to admission to $\mathrm{RHCU}(\%)$ & 96 \\
\hline \multicolumn{2}{|l|}{ Days from hospital admission to AGP (no. of patients) } \\
\hline$-1-3$ & 62.5 \\
\hline$-4-7$ & 27.8 \\
\hline$-\geq 8$ & 9.7 \\
\hline Average p02 prior to AGP (kPa) & 8.26 \\
\hline \multicolumn{2}{|l|}{ Survival based on CFS (\%) } \\
\hline-1 & 87 \\
\hline-2 & 55 \\
\hline-3 & 26 \\
\hline-4 & 10 \\
\hline Patients for escalation to ICU (\% total) & 50 \\
\hline - Patients admitted to ICU (\% total) & 20 \\
\hline ICU outcome (\% mortality) & 52 \\
\hline \multicolumn{2}{|l|}{ Novel treatment (\% of those eligible): } \\
\hline - Dexamethasone & 100 \\
\hline - Correct anticoagulation & 95 \\
\hline - Tocilizumab & 87 \\
\hline - Remdesivir & 61 \\
\hline RECOVERY trial recruitment (\%) & 29 \\
\hline Duration of hospitalisation (days) & 16.6 \\
\hline Average time on AGP (days) & 6.2 \\
\hline \multicolumn{2}{|l|}{ RHCU outcomes (\%) } \\
\hline - Discharged & 19 \\
\hline - Transferred to another ward & 22 \\
\hline - Transferred to ICU & 20 \\
\hline - Died & 39 \\
\hline Overall RHCU end hospital outcome (\% mortality) & 52 \\
\hline
\end{tabular}


Mean age was 66.2, 72\% male. 94\% white, in keeping with our population. Most common comorbidities included pre-existing lung disease (35\%), cardiovascular disease (33\%) and diabetes (29\%). 52\% had Rockwood clinical frailty score (CFS) $1-2$.

$50 \%$ were for full escalation. In total, $20 \%$ were transferred to Intensive Care Unit (ICU). Of those, average time from initiating AGP to ICU admission was 4 days. Patients transferred within 24 hours of initiating AGP and subsequently intubated, had a $40 \%$ mortality rate, versus $75 \%$ if transferred $>5$ days after AGP initiation. Overall ICU mortality rate was $52 \%$.

Average inpatient length was 16.6 days, mean AGP duration was 6.2 days. Patients with CFS 1 had a 13\% mortality following AGP initiation, versus 90\% mortality in those with CFS 4. We also noted a $1.1 \mathrm{kPa} \mathrm{PO} 2$ difference prior to AGP initiation for the survivors $(8.8 \mathrm{kPa}$ vs $7.7 \mathrm{kPa})$.

Overall RHCU outcome: 19\% discharged, 39\% died, 22\% transferred to other ward (87\% survived), 20\% ITU (48\% survived). ReSPECT form was completed in $87 \%$ and End of Life care-plan initiated in $81 \%$ who died.

Despite challenges, including staff shortage, low morale, oxygen and beds limitation, we remained up-to-date throughout the pandemic offering novel treatments. Of those eligible, $100 \%$ received dexamethasone, 95\% were appropriately anticoagulated, $87 \%$ were given tocilizumab and $61 \%$ received remdesivir. $29 \%$ of patients were recruited into the RECOVERY trial, far exceeding recruitment within the trust (11.2\%), regionally within the West Midlands (6.3\%), and nationally $(10 \%){ }^{1}$

\section{REFERENCE}

1. National Institute for Health Research. Recruitment progress report. RECOVERY. CPMS 45388, 02/3/2021.

\section{P216 COVID-19 AND ETHNICITY: HOW DOES IT IMPACT ADMISSION TO INTENSIVE CARE AND USE OF CPAP?}

A Krishna, R Naran, R Young, A Ainley. Barking, Havering and Redbridge University Trust, London, UK

\subsection{6/thorax-2021-BTSabstracts.325}

Background In the first wave of the COVID-19 pandemic, published data suggested that patients from ethnic minority backgrounds were disproportionately affected by the disease; however, there appears to be a paucity of data regarding specific outcomes such as admission to intensive care (ICU) and use of continuous positive pressure (CPAP) ventilation. ${ }^{1}$ We describe a patient cohort presenting to two urban district general hospitals, analysing whether ethnicity is associated with increased morbidity.

Methods A retrospective cohort analysis of 752 patients with a clinical or laboratory (RT-PCR positive) diagnosis of COVID-19 presenting to the respiratory units across two hospitals was conducted. Data was collected on patients' selfreported ethnic identity, admission to ICU, use of CPAP and intubation.

Results 48 patients' ethnic identities were unavailable and therefore excluded from analysis. Of the 704 patients included in analysis, median age was $61,58.1 \%$ male and $41.9 \%$ female. Within this cohort, non-white patients $(n=259)$ were more likely to require ICU admission and intubation when compared with white patients $(\mathrm{n}=445)(\mathrm{RR} 1.75$ [p <0.0015], RR 2.092 [p <0.0063]). Subgroup analysis showed, black patients $(n=47)$ were more likely to require ICU admission (RR 2.322 [p < 0.0184]), intubation (RR 3.293 [p <0.0017]) and CPAP (RR 1.612 [p <0.00183]) when directly compared to white patients. Patients of South Asian $(n=127)$ origin were more likely to require intubation (RR 1.98 [p <0.0396]) but no significant difference was noted in use of CPAP.

Conclusion Existing evidence suggests that patients from ethnic minority backgrounds were disproportionately affected by acute COVID-19 and are more likely to be exposed. Our analysis demonstrates that this patient group often experienced more severe disease requiring ICU admission and respiratory support, compared to white patients. Current hypotheses include a higher prevalence of comorbidities, socioeconomic factors and societal structural inequalities. Our study not only adds to current data and underlines the importance of continued research into this area, but also helps clinicians, particularly those within hospitals serving diverse populations, prepare resources for further potential surges of COVID-19.

\section{REFERENCE}

1. Sze S, et al. Ethnicity and clinical outcomes in COVID-19: a systematic Review and Meta-analysis. EClinicalMedicine 2020;100630.

\section{P217 IMPROVED COVID-19 OUTCOMES IN A LARGE NON- INVASIVE RESPIRATORY SUPPORT COHORT DESPITE NEW VARIANTS}

BML Porter, CD Turnbull, SB Evans, O Smith, R Lardner, RJ Hallifax, HV Bettinson, NP Talbot, M Bafadhel, NM Rahman, N Petousi. Oxford Centre for Respiratory Medicine, Oxford University Hospitals NHS Foundation Trust, Oxford, UK

\subsection{6/thorax-2021-BTSabstracts.326}

Background Respiratory high-dependency units (rHDU) are widely used to manage respiratory failure in coronavirus-19 (COVID-19) outside of the intensive care unit (ICU). Wave two variants of COVID-19 have been linked to increased rates of mortality and admission to ICU, however, their impact on a rHDU population, as well as the effects of new treatments, have not been previously studied. ${ }^{1}$ In this study we aimed to compare our clinical practice and rHDU outcomes between the two main UK waves of COVID-19 infection and identify factors that influenced outcomes in the second wave.

Method We conducted a single-centre, retrospective analysis of all patients with a diagnosis of COVID-19 admitted to the rHDU of our teaching hospital for respiratory support during the first wave from 23rd March to the 4th June 2020 and the second wave in between 10th October 2020 and 31st January 2021 when our evaluation ended. Patient data including virus genotype, treatments received and patient outcomes were collected and compared between waves.

Results In total, 348 patients were admitted to rHDU; 71 (20.4\%) during the first wave and 277 (79.6\%) in the second wave. Patient characteristics are shown in table 1. Mortality and intubation rates were lower in the second wave compared with the first. Patients in the second wave were less frail and more patients in the second wave received CPAP as their primary respiratory support and were able to prone. The VOC B.1.1.7 variant did not have a significant effect on rHDU outcome. 